\title{
A Proposed Inflammatory Score of Circulating Cytokines/ Adipokines Associated with Resistant Hypertension, but Dependent on Obesity Parameters
}

\author{
Ana Paula de Faria, ${ }^{10}$ Alessandra Mileni Versuti Ritter, ${ }^{10}$ Carolina Souza Gasparetti, ${ }^{1,2}$ Nathália Batista Corrêa, ${ }^{10}$ \\ Veridiana Brunelli, ${ }^{1 \oplus}$ Aurélio Almeida, ${ }^{1 \oplus}$ Nayara Fraccari Pires, ${ }^{1 \oplus}$ Rodrigo Modolo, ${ }^{3}$ Heitor Moreno Junior ${ }^{4}{ }^{\circledR}$ \\ Departamento de Farmacologia da Faculdade de Ciências Médicas da Universidade Estadual de Campinas, ${ }^{1}$ Campinas, SP - Brazil \\ Pontifícia Universidade Católica de Campinas (PUC-Campinas), ${ }^{2}$ Campinas, SP - Brazil \\ Departamento de Medicina Interna - Disciplina de Cardiologia da Faculdade de Ciências Médicas da Universidade Estadual de Campinas, ${ }^{3}$ \\ Campinas, SP - Brazil \\ Departamento de Medicina Interna da Faculdade de Ciências Médicas da Universidade Estadual de Campinas, ${ }^{4}$ Campinas, SP - Brazil
}

\begin{abstract}
Background: There is evidence that subclinical systemic inflammation is present in resistant hypertension (RHTN).

Objective: The aim of the study was to develop an integrated measure of circulating cytokines/adipokines involved in the pathophysiology of RHTN.

Methods: RHTN ( $n=112)$ and mild to moderate hypertensive $($ HTN) subjects $(n=112)$ were studied in a cross-sectional design. Plasma cytokines/adipokines (TNF-alpha, interleukins [IL]-6, -8, -10, leptin and adiponectin) values were divided into tertiles, to which a score ranging from 1 (lowest tertile) to 3 (highest tertile) was assigned. The inflammatory score (IS) of each subject was the sum of each pro-inflammatory cytokine scores from which anti-inflammatory cytokines (adiponectin and IL-10) scores were subtracted. The level of significance accepted was alpha $=0.05$.
\end{abstract}

Results: IS was higher in RHTN subjects compared with HTN subjects [4 (2-6) vs. 3 (2-5); $p=0.02$, respectively]. IS positively correlated with body fat parameters, such as body mass index $(r=0.40 ; p<0.001)$, waist circumference $(r=0.30 ; p<0.001)$ and fat mass assessed by bioelectrical impedance analysis $(r=0.31 ; p<0.001)$ in all hypertensive subjects. Logistic regression analyses revealed that IS was an independent predictor of $\operatorname{RHTN}(\mathrm{OR}=1.20 ; p=0.02)$, independent of age, gender and race, although it did not remain significant after adjustment for body fat parameters.

Conclusion: A state of subclinical inflammation defined by an IS including TNF-alpha, IL-6, IL-8, IL-10, leptin and adiponectin is associated with obese RHTN. In addition, this score correlates with obesity parameters, independently of hypertensive status. The IS may be used for the evaluation of conditions involving low-grade inflammation, such as obesity-related RHTN. Indeed, it also highlights the strong relationship between obesity and inflammatory process. (Arq Bras Cardiol. 2019; 112(4):383-389)

Keywords: Hypertension/physiopathology; Obesity; Inflammation; Cytokines; Adipokines; Probability; Risk Factors

\section{Introduction}

Inflammation is an important pathophysiological factor underlying hypertension, obesity, and metabolic syndrome. Overweight and obese status include a higher prevalence of hypertension and maladaptive consequences including cardiorenal and metabolic disorders. Excess visceral fat is a source of cytokines, that creates an inflammatory-oxidative stress cascade contributing to insulin resistance (IR), endothelial

Mailing Address: Ana Paula de Faria •

Rua Tessália Vieira de Camargo, 126. FCM 10. Universidade Estadual de Campinas (UNICAMP). Postal Code 13093-970, Barão Geraldo, Campinas, SP - Brazil

E-mail: aninha_cfaria@hotmail.com, ana.cabralfaria@gmail.com Manuscript received April 28, 2018, revised manuscript August 22, 2018, accepted September 05, 2018

DOI: $10.5935 / a b c .20190032$ dysfunction, vascular stiffening, and sodium retention in the kidney. ${ }^{1,2}$ The combined presence of obesity and IR also contributes to overactivation of both sympathetic nervous system (SNS) and the renin-angiotensin-aldosterone system. ${ }^{3}$ Ultimately, these disarrangements can lead to the occurrence of resistance to antihypertensive treatment. ${ }^{4}$

Our research group have explored inflammatory cytokines - the anti-inflammatory adiponectin and interleukin 10 and the pro-inflammatory leptin, tumor necrosis factor-alpha (TNF- $\alpha$ ), and interleukins 6 (IL-6) - in resistant hypertension (RHTN) associating them to the lack of blood pressure (BP) control and vascular-renal damage..$^{5-7}$ In addition, low-grade chronic inflammation, estimated by high C-reactive protein levels, was able to predict major fatal and nonfatal cardiovascular outcomes, and cardiac remodeling in this high-risk population. ${ }^{8-10}$

Adiponectin has an anti-inflammatory role and directly stimulates the production of nitric oxide (NO) in endothelial cells via phosphorylation of endothelial 
NO synthase..$^{10}$ It down regulates TNF- $\alpha$ production from macrophage by inhibiting nuclear transcription factor NF-kappa B. ${ }^{11,12}$ On the other hand, IL-6 inhibits adiponectin expression and secretion in 3T3-L1 adipocytes in vitro. ${ }^{13}$ Additionally TNF- $\alpha$ increases the secretion of leptin, ${ }^{14}$ which in turn stimulates the SNS. ${ }^{15}$ Since cytokines and adipokines have interconnected roles, we aimed with this study (1) to develop an integrated measure of several circulating cytokines/adipokines among subjects with RHTN and mild to moderate hypertension (HTN), and (2) to assess the potential impact of this inflammatory score (IS) on resistance to antihypertensive treatment.

\section{Population and methods}

A convenience sample of 112 subjects diagnosed with RHTN attending the Specialized Outpatient Clinic in RHTN of the University of Campinas (UNICAMP, Campinas, Brazil) and 112 HTN attending the Hypertension Clinic of Valinhos (Valinhos, Brazil) were consecutively enrolled in this cross-sectional study. RHTN was defined according to American Heart Association Statement as either (1) the subjects whose BP levels remain above goal $(\geq 140 / 90 \mathrm{mmHg}$ ) despite concurrent use of three or more antihypertensive drugs of different classes, or (2) those with controlled BP levels using four or more antihypertensive medication. Ideally, one of the agents should be a diuretic, and all agents should be prescribed at optimal doses. ${ }^{16}$ Patients with controlled BP using three or less antihypertensive drugs, or not yet controlled using two or less of these medications were classified as having HTN (grade 1 and grade 2 hypertension). ${ }^{17}$

A 6-month period follow-up for screening and exclusion of secondary causes of hypertension was performed to guarantee a precise diagnosis for HTN and "true" RHTN. The exclusion criteria were compounded with renal artery stenosis, coarctation of the aorta, pheochromocytoma, primary hyperaldosteronism (aldosterone to renin ratio $>20 \mathrm{ng} \cdot \mathrm{dL}^{-1}$ per ng. $\mathrm{mL}^{-1} \cdot \mathrm{h}^{-1}$ ), Cushing syndrome, obstructive sleep apnea-hypopnea syndrome (patients with previous polysomnographic diagnosis, or classified as high risk by the Berlin questionnaire). This period also included pill count to exclude the lack of BP control due to poor medication adherence, ${ }^{18}$ and ambulatory BP monitoring (ABPM) to exclude white coat hypertension. We also excluded patients with symptomatic ischemic heart disease, impaired renal function, chronic kidney disease (creatinine clearance $<30 \mathrm{~mL} / \mathrm{min} / 1.73 \mathrm{~m}^{2}$ ) and liver disease (medical history, and platelet and transaminase levels). Inclusion criterion was age over 18 years old.

\section{Blood pressure measurements}

Office systolic BP (SBP) and diastolic BP (DBP) were assessed by a trained health professional according to the European Society of Hypertension guidelines for the management of arterial hypertension. ${ }^{17}$ We used a validated digital sphygmomanometer (HEM-907XL, OMRON Healthcare Inc., Bannockburn, IL, USA). Ambulatory BP measurement was performed using an automatic oscillometric monitor (Spacelabs90207, SpacelabsInc, Redmon, WA, USA). Patients were instructed to maintain normal daily activities and record their 24-hour activities in a personal diary.

\section{Body composition}

The body composition was determined by the Bioimpedance Analyser 450 device (Biodynamics Corporation, Seattle, WA, USA) to assess fat-free mass and fat mass (FM). Briefly, the method is based on tetrapolar bioelectrical impedance (electrodes on feet and hands) to estimate mass and fluid compartments of the body. The measurements were performed after an 8-hour fast, and patients were instructed to avoid physical activity and smoking prior to the exam.

\section{Biochemical tests}

Blood samples were collected at morning after an 8-hour fast from patients in the sitting position. Plasma levels of aldosterone and renin were measured by radioimmunoassay (Immunotech SAS, Marseille, France), while the cytokines and adipokines - TNF-alpha, IL-6, IL-8, IL-10, leptin and adiponectin - were measured using enzyme-linked immunosorbent assay (ELISA) (R\&D Systems, Inc., Minneapolis, USA), according to the manufacturer's instructions. Creatinine clearance was calculated from 24h-urine creatinine level, urine flow rate, and plasma creatinine concentration as the removal rate per minute divided by plasma creatinine concentration.

\section{Statistical analyses}

Continuous variables were expressed as mean and standard deviation or median ( $1^{\text {st }}$ and 3 rd quartiles), according to data distribution assessed by the Kolmogorov-Smirnov test. Unpaired Student's t-test or the Mann Whitney test was applied to compare continuous data between the RHTN and HT. Categorical variables were presented in frequencies and percentages compared by chi-square or Fisher's exact test. Pearson or Spearman tests was used to assess correlation of continuous data. Multiple logistic regression analyses were performed to evaluate the association of IS with resistance to antihypertensive treatment, adjusting for potential confounders.

For IS calculation, the values of plasma cytokine/adipokine (TNF-alpha, IL-6, -8, -10, leptin and adiponectin) were divided into tertiles, and a score ranging from 1 (lowest tertile) to 3 (highest tertile) was assigned to them. The IS was considered as the sum of each pro-inflammatory cytokine score (TNF-alpha, IL-6, IL-8 and leptin) from which adiponectin and IL-10 both anti-inflammatory cytokines - scores were subtracted for each subject.

The analyses were performed using the software SigmaPlot (version 12, Systat Software, Inc., San Jose, CA USA, www. systatsoftware.com) and GraphPad Prism (version 7.00 for Windows, GraphPad Software, La Jolla, CA, USA, www.graphpad. com). The level of significance accepted was alpha 0.05.

\section{Results}

General characteristics of both hypertensive groups are described in Table 1. Body fat parameters (body mass index - BMI, waist circumference - WC and FM) revealed to be increased in the RHTN subjects, as well as lipid profile, glycated hemoglobin and aldosterone levels compared to their counterparts. Compared with HTN, RHTN individuals used 


\section{Original Article}

Table 1 - Clinical and biochemical characteristics of patients with mild-to-moderate hypertension (HTN) and patients with resistant hypertension (RHTN)

\begin{tabular}{|c|c|c|c|}
\hline & HTN $(n=112)$ & RHTN $(n=112)$ & $\mathrm{p}$-value \\
\hline Age (years) & $66 \pm 10$ & $58 \pm 10$ & $<0.001$ \\
\hline Female, $n(\%)$ & $63(56)$ & $78(70)$ & 0.27 \\
\hline Black, n (\%) & $13(12)$ & $55(49)$ & $<0.001$ \\
\hline $\mathrm{BMI}\left(\mathrm{Kg} / \mathrm{m}^{2}\right)$ & $27(25-31)$ & $31(27-35)$ & $<0.001$ \\
\hline$W C(\mathrm{~cm})$ & $94 \pm 12$ & $101 \pm 14$ & 0.003 \\
\hline FFM (Kg) & $53(46-62)$ & $55(49-64)$ & 0.11 \\
\hline $\mathrm{FM}(\mathrm{Kg})$ & $20(15-27)$ & $26(20-35)$ & $<0.001$ \\
\hline Office SBP (mmHg) & $139(131-149)$ & $149(134-163)$ & $<0.001$ \\
\hline Office DBP (mmHg) & $82(77-85)$ & $85(78-92)$ & 0.03 \\
\hline ABPM SBP (mmHg) & $126(118-134)$ & $130(118-144)$ & 0.03 \\
\hline ABPM DBP $(\mathrm{mmHg})$ & $75(70-81)$ & $75(70-86)$ & 0.22 \\
\hline $\mathrm{HR}$ (bpm) & $67(61-75)$ & $67(58-75)$ & 0.35 \\
\hline Glucose (mg/dL) & 97 (90-107) & $101(90-126)$ & 0.09 \\
\hline $\mathrm{HbA} 1 \mathrm{C}(\%)$ & $6.0(5.7-6.4)$ & $6.3(5.9-7.3)$ & 0.03 \\
\hline Cholesterol (mg/dL) & $165(136-187)$ & $181(150-209)$ & 0.001 \\
\hline LDL-c (mg/dL) & 88 (64-109) & $97(77-125)$ & 0.004 \\
\hline $\mathrm{HDL}-\mathrm{c}(\mathrm{mg} / \mathrm{dL})$ & $48(41-56)$ & $46(38-54)$ & 0.31 \\
\hline Triglycerides (mg/dL) & $108(80-150)$ & $126(93-185)$ & 0.02 \\
\hline Urea (mg/dL) & $34(27-43)$ & $35(27-44)$ & 0.52 \\
\hline Creatinine $(\mathrm{mg} / \mathrm{dL})$ & $0.95(0.79-1.10)$ & $0.94(0.80-1.18)$ & 0.19 \\
\hline Renin $(\mathrm{pg} / \mathrm{mL})$ & $29(14-73)$ & $25(12-72)$ & 0.39 \\
\hline Aldosterone $(\mathrm{pg} / \mathrm{mL})$ & $68(41-111)$ & $92(56-176)$ & 0.006 \\
\hline Creat. Clear $\left(\mathrm{mL} / \mathrm{min} / 1.73 \mathrm{~m}^{2}\right)$ & $75(58-93)$ & $81(61-97)$ & 0.89 \\
\hline
\end{tabular}

Values are expressed as mean \pm standard deviation or median (1st, 3rd quartiles), according to data distribution. BMI: body mass index; WC: waist circumference; FFM: fat-free mass; FM: fat mass; SBP: systolic blood pressure; DBP: diastolic blood pressure; ABPM: ambulatory blood pressure monitoring; HR: heart rate; HbA1C: glycated hemoglobin; LDL: low-density lipoprotein; HDL: high-density lipoprotein; Creat Clear: creatinine clearance.

a greater number of antiplatelet drugs and almost all classes of antihypertensive agents, except for angiotensin II receptor blockers (ARBs). On the other hand, a greater number of HTN subjects were taking statins (Table 2).

IS was higher in the RHTN compared to HTN group [4 (2-6) vs. 3 (2-5); $p=0.02$, respectively - Figure 1]. Curiously, IS positively correlated with BMI $(r=0.40 ; p<0.001)$, WC $(r=0.30 ; p<0.001)$ and FM $(r=0.31 ; p<0.001)$ in all hypertensive subjects.

Finally, the independent logistic regression models revealed that IS was associated with the presence of RHTN (Odds ratio $(\mathrm{OR})=1.20 ; p=0.02$ ), independently of age, gender and race, although it was no longer significant after the adjustments for the body fat parameters studied (Table 3).

\section{Discussion}

Our study revealed that the integrated measure of pro-inflammatory and anti-inflammatory cytokines/adipokines scores was associated with the occurrence of RHTN. The IS arises as a strong factor related to body fat parameters, suggesting the relevance of subclinical inflammation in obesity condition regardless of the hypertension degree.

Recent findings from our group have suggested that inflammatory process underlies the pathophysiology of RHTN and its related comorbidities like diabetes, obesity and metabolic syndrome. Altered levels of cytokines and adipokines, such as IL-10, IL-1 beta, adiponectin and leptin, were found in RHTN subjects compared to their controls. ${ }^{5,7,19}$ Hyperleptinemia and hypoadiponectinemia were associated with the lack of BP control, 5,19 as well as target organ damage - arterial stiffness and microalbuminuria - in this high-risk population. ${ }^{6}$ Obese diabetic RHTN subjects showed lower levels of adiponectin combined with a greater autonomic dysfunction (characterized by a hyperactive sympathetic system and a hypoactive parasympathetic system) than non-diabetic patients. ${ }^{20}$

Recently, we have found a huge prevalence of metabolic syndrome in these RHTN subjects (73\%), which may explain the high IS. Interestingly, the HTN group also showed a considerable prevalence of the syndrome $(60 \%),{ }^{21}$ which might justify the worsening of their score in our present study. 
Table 2 - Medications used by subjects with mild-to-moderate hypertension (HTN) and subjects with resistant hypertension (RHTN)

\begin{tabular}{|c|c|c|c|}
\hline & HTN $(n=112)$ & $\operatorname{RHTN}(n=112)$ & $\mathrm{p}$-value \\
\hline \multicolumn{4}{|l|}{ Antihypertensive drugs } \\
\hline Number of classes & $2(2-3)$ & $4(4-5)$ & $<0.001$ \\
\hline Diuretics, $\mathrm{n}(\%)$ & $70(63)$ & $108(96)$ & 0.02 \\
\hline ACEls, $n(\%)$ & $20(18)$ & $43(38)$ & 0.02 \\
\hline ARBs, $n(\%)$ & $81(72)$ & $61(54)$ & 0.01 \\
\hline CCBs, $n(\%)$ & $53(47)$ & $94(84)$ & $<0.001$ \\
\hline Beta-blockers, $\mathrm{n}(\%)$ & $14(13)$ & 79 (71) & $<0.001$ \\
\hline Central a-agonists, $n(\%)$ & $01(01)$ & $31(28)$ & $<0.001$ \\
\hline Statins, $n(\%)$ & $84(75)$ & $60(54)$ & 0.001 \\
\hline Glucose-lowering drugs, $n$ (\%) & $42(38)$ & $57(51)$ & 0.06 \\
\hline Antiplateletdrugs, $n(\%)$ & $20(18)$ & $65(58)$ & $<0.001$ \\
\hline
\end{tabular}

ACEls: angiotensin-converting enzyme inhibitors; ARBs: angiotensin receptor blockers; CCBs: calcium channel blockers.

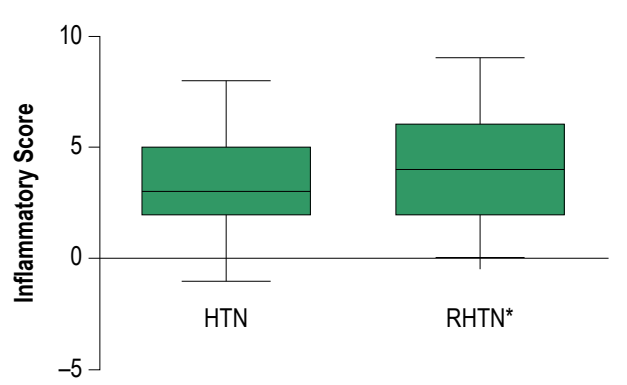

Figure 1 - Inflammatory score calculated between subjects with mild-to-moderate hypertension (HTN) and resistant hypertension (RHTN) $(3[2-5]$ vs. 4 [2-6], $p=0.02$, respectively). IS of each subject was the sum of each pro-inflammatory cytokine score (TNF-alpha, interleukins (IL) -6, -8, -10) from which the scores of anti-inflammatory cytokines (adiponectin and IL-10) were subtracted; ${ }^{*} p<0.05$ vs. HTN

In addition, the HTN group was older than the RHTN group, and hence an increased IS could be attributed to age. ${ }^{22}$

Experimental studies share similar findings of the role of inflammation on hypertension. Researchers have investigated changes in the systolic pressure of spontaneously hypertensive rats (SHR) treated with infliximab - a TNF-alpha-neutralizing agent. ${ }^{23}$ This study revealed cardiovascular benefits of inhibiting this cytokine in SHR with the reduction of both systolic BP and cardiac remodeling. The authors suggested a vasodilation dependent-mechanism in which the infliximab effect is able to induce the NO synthesis. ${ }^{23}$ Interestingly, a recent study ${ }^{24}$ described a new pathway of hypertension linked to an immune-inflammatory-oxidative stress cascade. Kirabo et al. ${ }^{24}$ demonstrated that an angiotensin II-infused mice model increased reactive oxygen species in dendritic cells releasing pro-inflammatory cytokines (IL-6, IL-1 beta, and IL-23), which in turn promoted $\mathrm{T}$ cell proliferation featuring a pro-inflammatory phenotype. Ultimately, these mechanisms led to hypertension, suggesting new potential targets to treat hypertension. ${ }^{24}$ Our results revealed that the IS - already investigated in type 2 diabetes $^{25}$ - was able to address in a single measure a great variety of mechanistically aligned cytokines/adipokines, involved in the pathophysiology of RHTN. Therefore, this approach could enhance estimation of the relation between the low-grade inflammation and high-risk populations such as the obese subjects with RHTN studied in this work.

It is well recognized that obesity, characterized by chronic activation of the immune system and inflammatory pathways, is a critical factor contributing to IR and type 2 diabetes, both comorbidities quite often presented in subjects with RHTN. In this context, many studies have supported this relationship. Esposito et al. ${ }^{26}$ found that weight loss and lifestyle changes decreased vascular inflammatory markers, such as IL-6, IL-18, and C-reactive protein, whereas adiponectin levels increased significantly in obese women. Similar effects of reducing levels of TNF-alpha were found in response to these interventions. ${ }^{27}$ Overweight and obesity have been suggested to cause microvascular dysfunction characterized by (1) impaired insulin sensitivity, (2) SNS activation and (3) increased vascular peripheral resistance. Along with this, changes in adipokines secretion leading to increased levels of free fatty acids and inflammatory mediators have also 


\section{Original Article}

Table 3 - Independent multiple logistic regressions to evaluate the association of the inflammatory score with the presence of resistant hypertension

\begin{tabular}{|c|c|c|}
\hline & $\mathrm{OR}(95 \% \mathrm{Cl})$ & $p$-value \\
\hline \multicolumn{3}{|l|}{ Model 1} \\
\hline IS & $1.20(1.02-1.38)$ & 0.02 \\
\hline \multicolumn{3}{|l|}{ Model 2} \\
\hline IS & $1.10(0.92-1.28)$ & 0.35 \\
\hline $\mathrm{BMI}\left(\mathrm{Kg} / \mathrm{m}^{2}\right)$ & $1.12(1.05-1.20)$ & $<0.01$ \\
\hline \multicolumn{3}{|l|}{ Model 3} \\
\hline IS & $0.97(0.80-1.18)$ & 0.73 \\
\hline WC (cm) & $1.04(1.01-1.07)$ & 0.01 \\
\hline \multicolumn{3}{|l|}{ Model 4} \\
\hline IS & $1.00(0.84-1.19)$ & 0.96 \\
\hline $\mathrm{FM}(\mathrm{Kg})$ & $1.08(1.04-1.13)$ & $<0.01$ \\
\hline
\end{tabular}

All models were adjusted for age, gender and race. IS: inflammatory score; BMI: body mass index; WC: waist circumference; FM: fat mass.

been suggested to be involved. ${ }^{28-30}$ Interestingly, impaired microvascular function in obese subjects was normalized one year after a gastric bypass surgery, and it was also associated with $\mathrm{BP}$ reduction. ${ }^{31}$ Elevated levels of free fatty acids lead to endothelial dysfunction by reducing the production of NO, and increasing endothelin-1 vasoconstrictor tone and the release of pro-inflammatory cytokines ${ }^{32}$ - which is an early hypertensionrelated factor associated with future cardiovascular events. ${ }^{33,34}$

Our findings showed that the association of IS and RHTN was abolished when the influence of body fat parameters was considered. Moreover, the IS was no longer significant after exclusion of obese subjects from both groups (data not shown). Our proposed score revealed its high dependence on obesity in the RHTN population, although this is expected since it is well-known these subjects are predominantly obese/ overweight, as we found in our study - prevalence of $88 \%$. Accordingly, the IS may reflect the inflammatory process underlying RHTN in an obesity-dependent manner, with the potential to be a clinical prognosis tool providing cardiovascular risk stratification in these obese subjects. On the other hand, we recognized that the design of this study is not sufficient to infer a temporal or cause-effect relationships. We also suggest that once obesity is established and hypertension is manifested, high BP may also contribute to further activation of inflammatory process. Thus, a vicious circle is created with both conditions - hypertension and obesity - that reinforces each other through inflammatory pathways.

Pharmacological or non-pharmacological treatments may affect inflammatory cytokines/adipokines. Studies have indicated that simvastatin reduces plasma levels of TNF-alpha and IL-6. ${ }^{35,36}$ Antihypertensive drugs such as candesartan, ${ }^{37}$ enalapril, ${ }^{38}$ and mineralocorticoid receptor antagonist have also been shown to reverse proinflammatory cytokines. ${ }^{39}$ Indeed, exercise and lifestyle modification reduced IL-8 levels in subjects with the metabolic syndrome, ${ }^{40}$ while significantly increased adiponectin levels in obese patients. ${ }^{26}$ Nevertheless, although these potential sources of variability may be present, they probably did not affect our findings since RHTN subjects had a high IS even though they used a greater number of antihypertensive agents. The use of individualized care also justifies the lack of standard therapy, and due to ethical issues, our subjects could not be assessed withdrawing the drugs. Finally, in a perspective of therapy approach, anti-inflammatory drugs or anticytokine molecules targeting the immune system, such as minocycline, can be attractive and of great interest in clinical setting to treat hypertension and prevent its cardiovascular complications, as supported by previous works. ${ }^{41-43}$

Some limitations should be mentioned. Since the population studied in this study is a convenience sample, with no sample size calculation, we recognize that our findings may not reflect the characteristics of the general population. Bias may also be present by comparing populations from different centers. It is worth mentioning that inflammatory process is quite complex and to measure its mediators is even more challenging since (i) it presents high costs, (ii) is still unavailable in clinical practice, and (iii) cutoff values may have heterogeneous profiles, which make the reproducibility more difficult. Even though, testing specificity and sensitivity in different populations are mandatory in order to guarantee a reliable score. Finally, this proposed score may change if the number of pro-inflammatory cytokines and/ or anti-inflammatory cytokines changes.

\section{Conclusion}

In conclusion, our findings suggest that the IS, addressing many circulating cytokines/adipokines, may provide clinically important information to complement cardiovascular risk stratification in obese RHTN subjects. Moreover, our proposed score seems to be highly dependent on obesity-related hypertension. It is necessary to validate this score in larger populations in order to allow its use safely in clinical practice.

\section{Author contributions}

Conception and design of the research and writing of the manuscript: de Faria AP; acquisition of data: de Faria AP, Ritter AMV; analysis and interpretation of the data and critical 
revision of the manuscript for intellectual content: de Faria $A P$, Ritter AMV, Gasparetti CS, Corrêa NB, Brunelli V, Almeida A, Pires NF, Modolo R, Moreno Junior $\mathrm{H}$; statistical analysis: de Faria AP, Modolo R; obtaining funding: de Faria AP, Ritter AMV, Moreno Junior $\mathrm{H}$.

\section{Potential Conflict of Interest}

No potential conflict of interest relevant to this article was reported.

\section{Sources of Funding}

This study was supported by the São Paulo Research Foundation (FAPESP), the National Council for Scientific and Technological Development (CNPq); and Coordination for the
Improvement of Higher Education Personnel (CAPES), Brazil.

\section{Study Association}

This study is not associated with any thesis or dissertation work.

\section{Ethics approval and consent to participate}

This study was approved by the Ethics Committee of the Faculdade de Ciências Médicas da Universidade Estadual de Campinas under the protocol number 188.161/2013; CAAE: 11189712.8.0000.5404. All the procedures in this study were in accordance with the 1975 Helsinki Declaration, updated in 2013. Informed consent was obtained from all participants included in the study.

\section{References}

1. Lyon CJ, Law RE, Hsueh WA. Minireview: adiposity, inflammation, and atherogenesis. Endocrinology. 2003;144(6):2195-200.

2. Aroor AR, McKarns S, Demarco VG, Jia G, Sowers JR. Maladaptive immune and inflammatory pathways lead to cardiovascular insulin resistance. Metabolism. 2013;62(11):1543-52

3. Briones AM, Aras-López R, Alonso MJ, Salaices M. Small artery remodeling in obesity and insulin resistance. Curr Vasc Pharmacol. 2014;12(3):427-37.

4. Hall ME, do Carmo JM, da Silva AA, Juncos LA, Wang Z, Hall JE. Obesity, hypertension, and chronic kidney disease. Int J Nephrol Renovasc Dis. 2014 Feb 18;7:75-88.

5. de Faria AP, Demacq C, Figueiredo VN, Moraes CH, Santos RC, Sabbatini AR, et al. Hypoadiponectinemia and aldosterone excess are associated with lack of blood pressure control in subjects with resistant hypertension. Hypertens Res. 2013;36(12):1067-72.

6. Sabbatini AR, Faria AP, Barbaro NR, Gordo WM, Modolo RG, Pinho C, et al. Deregulation of adipokines related to target organ damage on resistant hypertension. J Hum Hypertens. 2014;28(6):388-92.

7. Barbaro NR, Fontana V, Modolo R, De Faria AP, Sabbatini AR, Fonseca FH, et al. Increased arterial stiffness in resistant hypertension is associated with inflammatory biomarkers. Blood Press. 2015;24(1):7-13.

8. Cortez AF, Muxfeldt ES, Cardoso CR, Salles GF. Prognostic value of C-reactive protein in resistant hypertension. Am J Hypertens. 2016;29(8):992-1000.

9. Salles GF, Fiszman R, Cardoso CR, Muxfeldt ES. Relation of left ventricular hypertrophy with systemic inflammation and endothelial damage in resistant hypertension. Hypertension. 2007;50(4):723-8.

10. Chen H, Montagnani M, Funahashi T, Shimomura I, Quon MJ. Adiponectin stimulates production of nitric oxide in vascular endothelial cells. J Biol Chem. 2003;278(45):45021-6.

11. Ouchi N, Kihara S, Arita Y, Maeda K, Kuriyama H, Okamoto Y, et al. Novel modulator for endothelial adhesion molecules: adipocyte-derived plasma protein adiponectin. Circulation. 1999;100(25):2473-6.

12. Ouchi N, Kihara S, Arita Y, Okamoto Y, Maeda K, Kuriyama H, et al. Adiponectin, an adipocyte-derived plasma protein, inhibits endothelial NF-kappaB signaling through a cAMP-dependent pathway. Circulation. 2000;102(11):1296-301.

13. Fasshauer M, Kralisch S, Klier M, Lossner U, Bluher M, Klein J, et al. Adiponectin gene expression and secretion is inhibited by interleukin- 6 in 3T3-L1 adipocytes. Biochem Biophys Res Commun. 2003;301(4):1045-50.
4. Grunfeld C, Zhao C, Fuller J, PollackA, Moser A, Friedman I, et al. Endotoxin and cytokines induce expression of leptin, the ob gene product, in hamsters. J Clin Invest. 1996;97(9):2152-7.

15. Machleidt F, Simon P, Krapalis AF, Hallschmid M, Lehnert H, Sayk F. Experimental hyperleptinemia acutely increases vasoconstrictory sympathetic nerve activity in healthy humans. J Clin Endocrinol Metab. 2013;98(3):E491-6

16. Calhoun DA, Jones D, Textor S, Goff DC, Murphy TP, Toto RD, et al. Resistan hypertension: diagnosis, evaluation, and treatment. A scientific statement from the American Heart Association Professional Education Committee of the Council for High Blood Pressure Research. Hypertension. 2008;51(6):1403-19.

17. Mancia G, Fagard R, Narkiewicz K, Redon J, Zanchetti A, Böhm M, et al $2013 \mathrm{ESH} / \mathrm{ESC}$ guidelines for the management of arterial hypertension: the Task Force for the Management of Arterial Hypertension of the European Society of Hypertension (ESH) and of the European Society of Cardiology (ESC). Eur Heart J. 2013;34(28):2159-219.

18. de Souza WA, Sabha M, de Faveri Favero F, Bergsten-Mendes G, Yugar-Toledo $\mathrm{JC}$, Moreno $\mathrm{H}$. Intensive monitoring of adherence to treatment helps to identify "true" resistant hypertension. J Clin Hypertens (Greenwich). 2009;11(4):183-91.

19. de Haro Moraes C, Figueiredo VN, de Faria AP, Barbaro NR, Sabbatini AR Quinaglia T, et al. High-circulating leptin levels are associated with increased blood pressure in uncontrolled resistant hypertension. J Hum Hypertens. 2013;27(4):225-30.

20. Boer-Martins L, Figueiredo VN, Demacq C, Martins LC, Consolin-Colombo F, Figueiredo MJ, et al. Relationship of autonomic imbalance and circadian disruption with obesity and type 2 diabetes in resistant hypertensive patients. Cardiovasc Diabetol. 2011 Mar 22;10:24

21. Catharina AS, Modolo R, Ritter AMV, Sabbatini AR, Lopes HF, Moreno Junior H, Faria AP. Metabolic Syndrome-Related Features in Controlled and Resistant Hypertensive Subjects. Arq Bras Cardiol. 2018 Jun;110(6):514-521.

22. Enkhmaa B, Anuurad E, Zhang W, Kim K, Berglund L. Diverging trajectory patterns of systemic versus vascular inflammation over age in healthy Caucasians and African-Americans. Atherosclerosis. 2015;239(2):509-15.

23. Filho AG, Kinote A, Pereira DJ, Rennó A, dos Santos RC, Ferreira-Melo SE, et al. Infliximab prevents increased systolic blood pressure and upregulates the AKT/eNOS pathway in the aorta of spontaneously hypertensive rats. Eur J Pharmacol. 2013;700(1-3):201-9.

24. Kirabo A, Fontana V, de Faria AP, Loperena R, Galindo CL, Wu J, et al. DC isoketal-modified proteins activate T cells and promote hypertension. J Clin Invest. 2014;124(10):4642-56. 


\section{Original Article}

25. Daniele G, Guardado Mendoza R, Winnier D, Fiorentino TV, Pengou Z, Cornell J, et al. The inflammatory status score including IL-6, TNF-alpha, osteopontin, fractalkine, MCP-1 and adiponectin underlies whole-body insulin resistance and hyperglycemia in type 2 diabetes mellitus. Acta Diabetol. 2014;51(1):123-31.

26. Esposito K, Pontillo A, Di Palo C, Giugliano G, Masella M, Marfella R, et al. Effect of weight loss and lifestyle changes on vascular inflammatory markers in obese women: a randomized trial. JAMA. 2003;289(14):1799-804.

27. Monzillo LU, Hamdy O, Horton ES, Ledbury S, Mullooly C, Jarema C, et al. Effect of lifestyle modification on adipokine levels in obese subjects with insulin resistance. Obes Res. 2003;11(9):1048-54.

28. Scalia, R. The microcirculation in adipose tissue inflammation. Rev Endocr Metab Disord. 2013;14(1):69-76.

29. Bakker W, Sipkema P, Stehouwer CD, Serne EH, Smulders YM, van Hinsbergh $\mathrm{VW}$, et al. Protein kinase $\mathrm{C}$ theta activation induces insulin-mediated constriction of muscle resistance arteries. Diabetes. 2008;57(3):706-13.

30. Yudkin JS, Eringa E, Stehouwer CD. "Vasocrine" signalling from perivascular fat: a mechanism linking insulin resistance to vascular disease. Lancet. 2005;365(9473):1817-20.

31. Rossi M, Nannipieri M, Anselmino M, Pesce M, Muscelli E, Santoro G, et al. Skin vasodilator function and vasomotion in patients with morbid obesity: effects of gastric bypass surgery. Obes Surg. 2011;21(1):87-94 .

32. Vincent MA, Montagnani M, Quon MJ. Molecular and physiologic actions of insulin related to production of nitric oxide in vascular endothelium. Curr Diab Rep. 2003;3(4):279-88.

33. Landmesser U, Drexler H. Endothelial function and hypertension. Curr Opin Cardiol. 2007;22(4):316-20.

34. Perticone F, Ceravolo R, Pujia A, Ventura G, lacopino S, Scozzafava A, et al. Prognostic significance of endothelial dysfunction in hypertensive patients. Circulation. 2001;104(2):191-6.
35. Koh KK, Son JW, Ahn JY, Jin DK, Kim HS, Choi YM, et al. Comparative effects of diet and statin on NO bioactivity and matrix metalloproteinases in hypercholesterolemic patients with coronary artery disease. Arterioscler Thromb Vasc Biol. 2002;22(9):e19-23.

36. Koh KK, Schenke WH, Waclawiw MA, Csako G, Cannon RO 3rd. Statin attenuates increase in $\mathrm{C}$-reactive protein during estrogen replacement therapy in postmenopausal women. Circulation. 2002;105(13):1531-3.

37. Koh KK, Ahn JY, Han SH, Kim DS, Jin DK, Kim HS, et al. Pleiotropic effects of angiotensin II receptor blocker in hypertensive patients. J Am Coll Cardiol. 2003;42(5):905-10.

38. Trevelyan J, Brull DJ, Needham EW, Montgomery HE, Morris A, Mattu RK. Effect of enalapril and losartan on cytokines in patients with stable angina pectoris awaiting coronary artery bypass grafting and their interaction with polymorphisms in the interleukin-6 gene. Am J Cardiol. 2004;94(5):564-9.

39. Guo C, Ricchiuti V, Lian BQ, Yao TM, Coutinho P, RomeroJR, etal. Mineralocorticoid receptor blockade reverses obesity-related changes in expression of adiponectin, peroxisome proliferator-activated receptor-gamma, and proinflammatoryadipokines. Circulation. 2008;117(17):2253-61.

40. Trøseid M, Lappegård KT, Claudi T, Damås JK, Mørkrid L, Brendberg R, et al. Exercise reduces plasma levels of the chemokines MCP-1 and IL-8 in subjects with the metabolic syndrome. Eur Heart J. 2004;25(4):349-55.

41. Hu P, ThinschmidtJS, Yan Y, Hazra S, Bhatwadekar A, Caballero S, et al. CNS inflammation and bone marrow neuropathy in type 1 diabetes. Am J Pathol. 2013;183(5):1608-20.

42. Santisteban MM, Ahmari N, Carvajal JM, Zingler MB, Qi Y, Kim S, et al. Involvement of bone marrow cells and neuroinflammation in hypertension. Circ Res. 2015;117(2):178-91.

43. Liu W, Wang X, Feng W, Li S, Tian W, Xu T, et al. Lentivirus mediated IL-17R blockade improves diastolic cardiac function in spontaneously hypertensive rats. Exp Mol Pathol. 2011;91(1):362-7. 